\title{
Vertical jump and agility performance improve after an 8-week conditioning program in youth female volleyball athletes
}

\author{
DAVIS HALE ${ }^{1}$, ROGER KOLLOCK ${ }^{2}$, JEFF PACE ${ }^{3}$, GABE SANDERS $^{4}$ \\ ${ }_{1,2}$ Department of Kinesiology and Rehabilitative Sciences, The University of Tulsa, USA \\ ${ }^{3}$ Titan Sports and Performance Center, USA \\ ${ }^{4}$ Department of Kinesiology and Health, Northern Kentucky University, USA
}

Published online:March 31, 2019

(Accepted for publication March 12, 2019)

DOI:10.7752/jpes.2019.01109

\begin{abstract}
:
The purpose of this study was to determine if an 8-week combined strength and plyometric/agility offseason conditioning program improved performance on three vertical jump protocols and agility time in youth female volleyball athletes. The current study was an 8 -week prospective cohort repeated measures experimental design. Eleven female youth volleyball athletes (age $15.1 \pm 2.7$ ) completed an 8-week mesocycle offseason conditioning program that consisted of two separate blocks (4-weeks each) of conditioning (strength and plyometric/agility). The first 4 -weeks of conditioning included $2 \mathrm{x}$ week (60 min each) of strength building exercises (weeks 1-4). The second 4-weeks of conditioning included $2 \mathrm{x}$ week (60 min each) of power based ploymetric and agility exercises (weeks 5-8). All four variables significantly improved from pre- and posttesting periods. The three vertical jump protocols significantly increased in height (BVJ: 37.1 vs 40.9, p.000; CMJ: 43.9 vs $46.7, p .000$; AVJ: 53.3 vs $58.7, p .001)$ with medium effect sizes. In addition, the agility times decreased following the 8 -weeks of offseason conditioning (9C: 25.3 vs $23.6, p .000)$ with a large effect size $(d=$ .871). The average improvement across the four variables was $7.7 \%$.

Key words: youth, plyometrics, agility, strength training
\end{abstract}

\section{Introduction}

Unique performance related attributes for the sport of volleyball include speed, maximal vertical jump, frequent change-of-direction, and repeated over-head movements (Kleinwolterink, Moody, \& Mayhew, 2010; Martinez, 2017). Volleyball athletes must employ specific movement patterns with high levels of force, high rates of speed, and quick changes-of-direction (Paz et al., 2017; J. M. Sheppard, Gabbett, \& Stanganelli, 2009; Stanganelli, Dourado, Oncken, Mançan, \& da Costa, 2008). Reeser and Bahr reported that volleyball athletes may perform between 30,000 and 40,000 jumping movements in a single year (Reeser \& Bahr, 2017). In addition to total jump, athlete have been found to engage in greater than 100 high intensity jumps throughout practice and games, along with the other volleyball specific movements that rely predominately on the anaerobic energy pathways with partial to complete recovery between efforts (Sanders, Boos, Shipley, Scheadler, \& Peacock, 2018; J. M. Sheppard et al., 2009; VanHeest, 2003).

Of these anaerobic efforts, jumping ability (approach and block) account for almost half of the total game activities placing an increased demand on the ability to produce high amounts of lower body anaerobic power repeatedly (Martinez, 2017; Voigt \& Vetter, 2003). According to Marques and colleagues, the ability to generate high amounts of lower body anaerobic power is dependent on the sport specific conditioning program that should incorporate exercises targeting strength and power development (Marques, Tillaar, Vescovi, \& Gonzalez-Badillo, 2008). In addition, recently Gjinovci et al. discovered that plyometric based conditioning improved performance outcomes in female volleyball players including the countermovement jump and standing broad jump ability (Gjinovci, Idrizovic, Uljevic, \& Sekulic, 2017). The authors concluded that plyometric training twice per week improved the athletes' jumping ability more than skill based conditioning alone. However, both previously mentioned studies did not include younger female volleyball athletes under the age of 18 enrolled in an offseason conditioning program.

In addition to jumping performance, volleyball requires athletes to repeatedly change directions which emphasizes the need for agility based training for optimal performance (Gabbett \& Georgieff, 2007). Within the context of volleyball, Barnes and colleagues suggested that agility be defined as the "ability to change direction with minimal loss of control and/or average speed" (Barnes et al., 2007). These researchers found a strong correlation between agility performance and jumping ability in Division I female volleyball athletes. Agility performance has also been determined as a predictor of success in repeated jumping and sprinting abilities across the various volleyball positions (Sahin, 2014; J. Sheppard et al., 2008; J. M. Sheppard et al., 2009). Conditioning programs designed to address agility performance should include activities that train the 
neuromuscular capacity of multidirectional court movements such as power based and plyometric type exercises (Fathi et al., 2018; Kleinwolterink et al., 2010).

Incorporating both strength and plyometric exercises to improve jumping and agility performance has demonstrated promise in youth athletic populations (Faigenbaum et al., 2007). Recently Lloyd and colleagues reported adolescent athletes respond more favorably to a combined strength and power conditioning program than a traditional strength program alone (Lloyd et al., 2013). However, the combined effect of a strength and plyometric based conditioning program on jumping and agility performance on adolescent female volleyball players in lacking (Pereira, M Costa, Santos, Figueiredo, \& Vicente João, 2015; Voelzke, Stutzig, Thorhauer, \& Granacher, 2012). The most recent study on a combined strength and plyometric training program in adolescent volleyball players was conducted by Fathi et al., and concluded a combined program yields safe and significant improvements in sprint and jumping outcomes (Fathi et al., 2018). However, the previous study only used male adolescent athletes.

Volleyball sport specific vertical jumping ability can be assessed through different types of vertical jumping protocols such as the block vertical jump (BVJ), the countermovement vertical jump (CMJ), and the two-step approach vertical jump (AVJ) (Martinez, 2017; Mayberry, Patterson, \& Wagner, 2018; Reeser \& Bahr, 2017; Yingling et al., 2018). Sport specific agility testing for volleyball can be assessed with a 9-cone (9C) test to determine the athletes' ability to generate quick and accurate movement with change-of-direction and/or acceleration and deceleration (Lloyd et al., 2013). Thus, the purpose of this study was to determine if an 8-week combined strength and plyometric/agility offseason conditioning program improved performance on three vertical jump protocols and agility time in youth female volleyball athletes. The researchers hypothesized that all four variables would improve following the 8-week offseason conditioning program.

\section{Material \& methods}

The current study was an 8-week prospective cohort repeated measures experimental design. Following IRB approval, youth female volleyball athletes were recruited for study participation upon enrollment in an offseason conditioning program at a sport specific training facility. The offseason conditioning program consisted two training session per week for 8-weeks that consisted of strength, agility, and ploymetric based training. All participants were tested prior (pre) to and at the end (post) of the 8-week conditioning program in four areas of performance that included the block vertical jump (BVJ), countermovement vertical jump (CMJ), approach vertical jump (AVJ), and 9-cone agility time (9C). The inclusion of three different measures of vertical jump has been previously recommended to determine current athlete strengths and limitations (Martinez, 2017; Sattler, Sekulic, Hadzic, Uljevic, \& Dervisevic, 2012). It was hypothesized that all four variables would improve from the pre- and post- testing periods due to the 8-week conditioning program. The variables measured in this study included 3 types of vertical jumps (BVJ, CMJ, AVJ), one measure of agility (9C), and height/weight. Prior to each biweekly conditioning session and each testing session (pre-, post-), athletes completed a 15-minute standardized warm-up consisting of general and dynamic movements. Testing and conditioning sessions were performed in an indoor facility within a thermoneutral environment. Both testing sessions occurred separately and prior to any conditioning/practices sessions on that particular day. Athletes were instructed not to be involved in strenuous exercises for at least 48 hours before the testing sessions and to consume their normal pretraining diet. Both the pre- and post- testing sessions were scheduled on a Monday because athletes had rested through the weekend. The pretesting session began with height, weight, and reach height measurements. The athletes were then familiarized with the three different vertical jumps and agility tests.

\section{Participants}

A total of 15 female youth volleyball athletes enrolled in an 8-week offseason conditioning program. Of the 15 who started the program, 11 completed the entire 8-week program with included pre- and post- testing periods (age 15.1 \pm 2.7$)$. All participants included in this study $(n=11)$ started and completed the project free from injury and illness. Participants did not have a history of injury or orthopedic limitation that might impair their ability to complete the prescribed exercises. Prior to study participation, all players and their guardians provided the appropriate consent and assent. The study was approved by the participating university's institutional review board and was declared ethical for participation. The 11 study participants were only enrolled in the 8-week prescribed offseason conditioning program during the study duration. No additional conditioning was allowed outside of the prescribed 8-week period. Descriptive data for the volleyball athletes is presented in Table 1.

Table 1. Descriptive characteristics of athletes*

\begin{tabular}{|l|l|l|l|}
\hline Age (y) & Height $(\mathbf{c m})$ & Weight $\mathbf{( k g )}$ & Standing reach height $(\mathbf{c m})$ \\
\hline $15.1 \pm 2.7$ & $173.2 \pm 3.3$ & $65.1 \pm 6.7$ & $215.6 \pm 6.8$ \\
\hline & ? Data reported as mean \pm SD. \\
\hline
\end{tabular}


Vertical Jump Assessment

To assess vertical jump, each athlete completed three different vertical jump protocols (BVJ, CMJ, AVJ). Three testing stations were assigned by researchers. The athletes were randomly assigned to the order in which they completed the three vertical jumping tests. Prior to pretesting, athlete standing reach height was obtained to the nearest half inch while wearing their normal volleyball footwear with the athletes instructed to stand with their feet flat on the ground and fully extend their dominant arm and hand. All vertical jump measures were completed using the Vertec (JUMPUSA.com, Sunnyvale, CA, USA) vertical jump testing device. Three trials were allowed for each of the three vertical jump stations with the best of three (nearest half $\mathrm{cm}$ ) retained for statistical analysis. Athletes were instructed to wait 3-5 minutes between each of the three vertical jump testing stations.

The BVJ is a reliable vertical jump test designed to assess jumping ability from a defensive position (Martinez, 2017). Test-retest reliability in this sample for the BVJ was acceptable $(\alpha=.983)$. The athletes were instructed to perform the jump from a defensive position with hands positioned in front of the chest. While in this position, athletes perform a countermovement jump without arm swing that is typical for the sport specific practices or games. The jump is performed with the athlete attempting to reach as high as possible with arm in fully extended position. The CMJ is a reliable (Martinez, 2017; Sattler et al., 2012) vertical jump test used to measure jump height with a downward movement followed by a jump for maximum height. Test-retest reliability in this sample for the CMJ was acceptable $(\alpha=.991)$. The CMJ began with the athlete standing in the upright position followed by a fast downward movement close to $90^{\circ}$ knee flexion immediately followed by an upward jump with full arm swing. The AVJ is a reliable (Martinez, 2017; Sattler et al., 2012) vertical jump test designed to measure jumping ability with a countermovement after a 3-step approach. Test-retest reliability in this sample for the AVJ was acceptable $(\alpha=.925)$. The AVJ began with the athlete starting 3-steps from the testing device followed by a half-drop jump (countermovement) with arm swing progressing to a maximum jump for height.

\section{Agility Assessment}

To assess agility, a 9-cone test (Figure 1) with movement starting to the right was used. Test-retest reliability of the 9C in this sample was acceptable $(\alpha=.928)$. The $9 \mathrm{C}$ agility test is used to determine the ability of the athletes to repeatedly turn and change directions. Athletes completed two trials of the 9C agility test with the best time retained for statistical analysis (nearest hundredth of a second). The test started with the athlete starting in a 2-point stance in a static position behind the center cone with time starting on the first movement. The athlete then sprinted to touch cone 1 (with either hand) and returned back to the center cone touching with either hand. The athlete continued this pattern on cones 3-8 moving in a clockwise direction with the time stopping once the athlete touched the center cone after returning back from cone 8 .

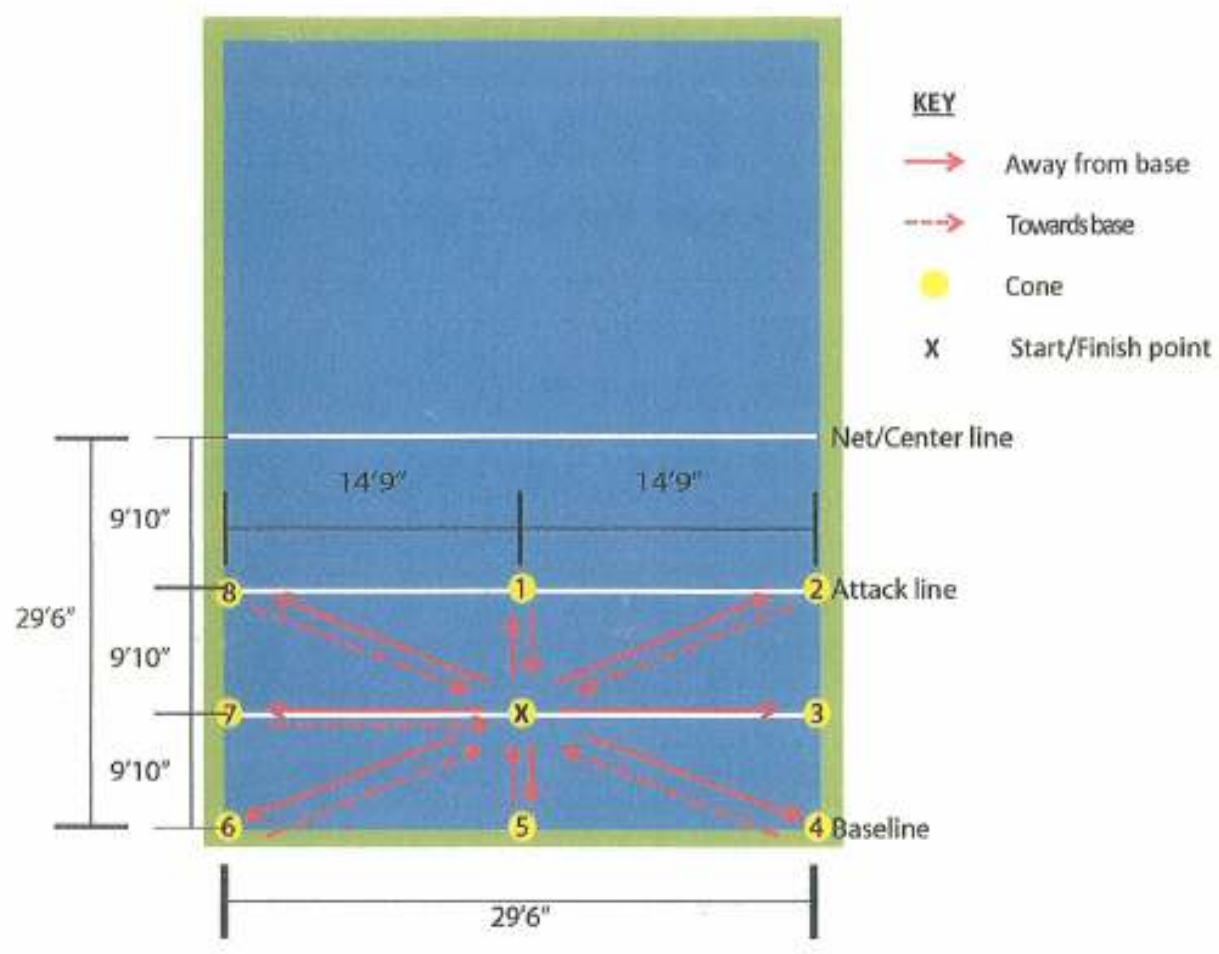

Fig. 1. 9-cone agility test 


\section{8-Week Conditioning Program}

Eleven female youth volleyball athletes completed an 8-week mesocycle offseason conditioning program (Tables $2 \& 3$ ). The 8-week offseason conditioning program consisted of two separate blocks (4-weeks each) of conditioning (strength and plyometric/agility). The strength building phase of conditioning occurred prior to the plyometric/agility phase of conditioning to ensure athletes obtained adequate strength as recommended by Faigenbaum and colleagues (Faigenbaum et al., 2007). The first 4-weeks of conditioning included $2 \mathrm{x}$ week (60 min each) of strength building exercises (weeks 1-4). The second 4-weeks of conditioning included $2 \mathrm{x}$ week (60 min each) of power based ploymetric and agility exercises (weeks 5-8). Three vertical jump protocols (BVJ, CMJ, AVJ) and an agility test (9C) were administered at the beginning of the first week and at the end of week 8 of the offseason conditioning program.

Athletes reported to the sport specific training facility twice weekly to complete the prescribed conditioning sessions. A 15-minute standardized warm-up preceded all conditioning sessions consisting of general and dynamic movements. Each training session ended with a 5-minute cool-down consisting of dynamic and static stretching. The athletes were instructed not to be enrolled in any other conditioning program other than sport specific practices during the 8-week offseason conditioning program. The first block of the mesocycle (Table 2) included two days per week of 4-weeks of strength based exercises including bodyweight, machine, and implement based resistance exercises. Athletes completed 2-5 sets of each exercise with 10-30 repetitions depending on exercise type. The second block of the mesocycle (Table 3) included two days per week of 4weeks of plyometrics, interval, and agility exercises including sled push, Vertimax jumps, shuttle run, crunches and planks, multidirectional hops, and box jumps. The power based and agility exercises included 3-5 sets of 610 repetitions based on the exercise type. The interval exercises included 3 sets of a 1:2 work rest ratio of 20-40 seconds.

Table 2. Strength training program (weeks 1-4)

\begin{tabular}{ll}
\hline Goal: upper and lower body strength \\
\multicolumn{2}{l}{ Sessions 1-8 (Monday and Thursday) } \\
\hline Monday & Thursday \\
Shuttle squats & Kettlebell deadlifts \\
TRX incline row & Dumbbell rows \\
Kettlebell swings & Single leg squats \\
Incline pushups & Lateral raises with band \\
Lateral lunges & TRX hamstring curls \\
High pull with band & Swimmers with band \\
Medicine ball power ups & Single leg hip extension \\
Versa Climber & Bosu ball crossover crunch \\
Air bike & Glute/ham bench \\
Rower & \\
Prone plank & \\
Supine plank & \\
\hline
\end{tabular}

Table 3. Plyometric/agility training program (weeks 5-8)

\begin{tabular}{ll}
\hline \multicolumn{2}{l}{ Goal: lower body power/agility } \\
Sessions 1-8 (Monday and Thursday) \\
Monday & Thursday \\
\hline Sled push & Multidirectional hops \\
Vertimax max jumps & Approach box jumps \\
Versa climber & Vertimax max jumps \\
Air bike & Speed skater \\
Shuttle run & Rower \\
TRX knee tucks & Big wave \\
Abdominal crunches & Lateral planks \\
\hline
\end{tabular}

\section{Statistical Analysis}

The Statistical Package for the Social Sciences SPSS (v24, IBM Corp., Chicago, IL, USA) was used for the statistical analyses. Descriptive data was calculated and is presented in Table 1. Prior to data comparisons, a Kolmogorov-Smirnov test of normality was performed for each of the four variables and was determined to be from a normal distribution (BVJ: $p=.096, \mathrm{CMJ}: p=.200, \mathrm{AVJ}: p=.187,9 \mathrm{C}: p=.127$ ). A series of paired sample t-tests were performed to compare pretest and posttest vertical jump heights and agility times with post hoc effect size calculations utilizing Cohen's $d$ (Harmandeep, Satinder, Amita, \& Anupriya, 2015). The significance level was set at .05. 


\section{Results}

All four variables significantly improved from pre- and post- testing periods. The three vertical jump protocols significantly increased in height (BVJ: 37.1 vs 40.9, p.000; CMJ: 43.9 vs 46.7, p.000; AVJ: 53.3 vs $58.7, p .001$ ) with medium effect sizes (Table 4). In addition, the agility times decreased following the 8-weeks of offseason conditioning (9C: 25.3 vs $23.6, p .000)$ with a large effect size $(d=.871)$. The average improvement across the four variables was $7.7 \%$.

Table 4. Mean and SD results from pre- and post- testing

\begin{tabular}{llllll}
\hline Tests & Pretest & Posttest & $\boldsymbol{p}$ & $\mathbf{\%}$ & $\boldsymbol{d}$ \\
\hline BVJ $(\mathrm{cm})$ & $37.1 \pm 6.4$ & $40.9 \pm 6.1$ & $0.00^{*}$ & 9.31 & 0.612 \\
CMJ $(\mathrm{cm})$ & $43.9 \pm 6.4$ & $46.7 \pm 6.9$ & $0.00^{*}$ & 5.97 & 0.422 \\
AVJ $(\mathrm{cm})$ & $53.3 \pm 7.4$ & $58.7 \pm 7.1$ & $0.001^{*}$ & 9.10 & 0.736 \\
9C $(\mathrm{s})$ & $25.3 \pm 1.9$ & $23.6 \pm 2.0$ & $00.00^{*}$ & 6.71 & 0.871 \\
\hline BVJ = block vertical jump; CMJ = countermovement vertical jump; AVJ = approach vertical jump; 9C $=9-$ \\
cone agility test; \% = percentage difference between testing periods; $d=$ effect size \\
* Significantly different at $p \leq 0.05$.
\end{tabular}

\section{Discussion}

The current study investigated the effect of an 8-week offseason strength and plyometric/agility based conditioning program on vertical jumping and agility performance in adolescent female volleyball athletes. Results indicate a significant improvement in three vertical jumping protocols and agility times following the combined plyometric and strength based conditioning program. The percentage increase in vertical jump heights were BVJ: $9.31 \%$, CMJ: 5.97\%, and AVJ: 9.1\% with medium effect sizes. The percentage decrease in agility times (9C) was $6.71 \%$ with a large effect size.

The overall improvements in jumping ability are similar to findings by Harmandeep et al. in a 2015 study with elite adult male volleyball athletes (Harmandeep et al., 2015). In that study, the researchers determined that a 6 -week plyometric only training program significantly increased vertical jump height by $7.5 \%$. Marques and colleagues (Marques et al., 2008) also reported significant improvements in an off-season plyometric conditioning program for professional female volleyball athletes. Those athletes participated in a 12week combined plyometric and strength based conditioning program along with skill based practices. Overall jumping ability significantly increased following the 12-week condition program on average by $9.7 \%$. The average vertical jump height increase across the three vertical jumping protocols in the current study was $8.1 \%$. While the current study and the Marques study included female volleyball athletes, the current study included a younger subset of females (15 year old versus 25 years old). Findings from both studies reported no injuries occurred during the conditioning programs confirming that vertical jumping ability can improve safely within a younger female athlete population.

A previously mentioned study that investigated the effect of a plyometric condition program on jumping ability was a 2017 study by Gjinovci and colleagues (Gjinovci et al., 2017). That study included 41 elite female volleyball players with an average age of 21 years divided into a skill-based conditioning only and plyometric conditioning only group. The athletes completed 12-weeks of a twice per week plyometric conditioning program or a skill-based conditioning program during a competitive season with various performance parameters assessed before and after the conditioning program. Results indicated that the plyometric conditioning group increased vertical jump heights by $21 \%$ versus $15 \%$ in the skill-based conditioning group. Such large improvements may be the result of the higher level athletes included in this study. Regardless, it appears that plyometric based conditioning yields significant vertical jumping improvement in various populations versus lack of plyometric conditioning.

Regarding younger female athletes, researchers Pereia and colleagues (Pereira et al., 2015) also reported improvement in vertical jumping ability following an 8-week plyometric conditioning program. Their study included adolescent female volleyball athletes (13.9 years old) participating in a competitive season along with completing the twice per week plyometric conditioning program. On average, the athletes improved jumping height (countermovement jump) by $16.7 \%$ following the 8 -week plyometric conditioning program. Of note, the authors determined that future studies include vertical jumping tests that measure blocking and spiking abilities. Evidence from a 2010 study (Kleinwolterink et al., 2010) with female volleyball players also reported an increase in vertical jumping performance following a 6-week speed-jump conditioning program. The authors noted an average increase in vertical jump heights of 5.9\%-14\% following the 6 -week conditioning program. The current study included three vertical jumping tests, specifically the ability to block (BVJ) and spike (AVJ) and saw significant increases following the prescribed 8-week combined plyometric and strength based conditioning program.

Voelzke and colleagues (Voelzke et al., 2012) investigated the impact of a 5-week combined strength and plyometric conditioning program on jumping and agility performance. Eight elite national level female volleyball athletes (23.8 years old) completed the combined 5-week strength and plyometric conditioning program during the last mesocycle in the preseason. Training sessions were scheduled twice per week along 
with their regular scheduled 4-5 skill-based practice sessions per week. Conclusions drawn from this study included a slight increase in vertical jump height $1.6 \%$ and agility time decrease by $3.8 \%$. The authors report that smaller changes were experienced than in previous studies due to their athletes already being at an elite state of conditioning during program enrollment. In the current study, agility times decreased $6.7 \%$ following the 8 week combined strength and plyometric conditioning program.

\section{Conclusions}

Results from the current study indicated significant improvements in vertical jump heights and agility times following an 8-week combined strength and plyometric/agility conditioning program confirming the researchers' hypothesis. Even though these findings were statistically significant, the authors note several recommendations for future research. Future studies should include a larger sample of youth female volleyball athletes enrolled in an offseason conditioning program. Previous studies with similar athletes also excluded a control group and occasionally contained a second group completing skill-based conditioning only. Future research is recommended testing the impact of plyometric only versus strength training only in improving vertical jump and agility efforts in youth female volleyball athletes

\section{Conflicts of interest}

The authors declare no conflict of interest.

\section{Acknowledgments}

The authors would like to acknowledge Titan Sports and Performance for their assistance with programming and testing. This work was supported by the University of Tulsa Faculty Development Summer Fellowship.

\section{References}

Barnes, J. L., Schilling, B. K., Falvo, M. J., Weiss, L. W., Creasy, A. K., \& Fry, A. C. (2007). Relationship of jumping and agility performance in female volleyball athletes. Journal of strength and Conditioning Research, 21(4), 1192.

Faigenbaum, A. D., McFarland, J. E., Keiper, F. B., Tevlin, W., Ratamess, N. A., Kang, J., \& Hoffman, J. R. (2007). Effects of a short-term plyometric and resistance training program on fitness performance in boys age 12 to 15 years. Journal of sports science \& medicine, 6(4), 519.

Fathi, A., Hammami, R., Moran, J., Borji, R., Sahli, S., \& Rebai, H. (2018). Effect of A 16 Week Combined Strength and Plyometric Training Program Followed by A Detraining Period on Athletic Performance in Pubertal Volleyball Players. Journal of strength and Conditioning Research.

Gabbett, T., \& Georgieff, B. (2007). Physiological and anthropometric characteristics of Australian junior national, state, and novice volleyball players. Journal of strength and Conditioning Research, 21(3), 902.

Gjinovci, B., Idrizovic, K., Uljevic, O., \& Sekulic, D. (2017). Plyometric training improves sprinting, jumping and throwing capacities of high level female volleyball players better than skill-based conditioning. Journal of sports science \& medicine, 16(4), 527.

Harmandeep, S., Satinder, K., Amita, R., \& Anupriya, S. (2015). Effects of six-week plyometrics on vertical jumping ability of volleyball players. Research Journal of Physical Education Sciences, 2320, 9011.

Kleinwolterink, A., Moody, S., \& Mayhew, J. (2010). Effect of speed-jump training on volleyball specific measurements skills in female athletes. The Journal of Strength \& Conditioning Research, 24, 1.

Lloyd, R. S., Read, P., Oliver, J. L., Meyers, R. W., Nimphius, S., \& Jeffreys, I. (2013). Considerations for the development of agility during childhood and adolescence. Strength \& Conditioning Journal, 35(3), 211.

Marques, M. C., Tillaar, R., Vescovi, J. D., \& Gonzalez-Badillo, J. J. (2008). Changes in strength and power performance in elite senior female professional volleyball players during the in-season: a case study. $J$ Strength Cond Res, 22(4), 1147-1155. doi:10.1519/JSC.0b013e31816a42d0

Martinez, D. B. (2017). Consideration for Power and Capacity in Volleyball Vertical Jump Performance. Strength \& Conditioning Journal, 39(4), 36-48.

Mayberry, J. K., Patterson, B., \& Wagner, P. (2018). Improving vertical jump profiles through prescribed movement plans. The Journal of Strength \& Conditioning Research, 32(6), 1619-1626.

Paz, G. A., Gabbett, T. J., Maia, M. F., Santana, H., Miranda, H., \& Lima, V. (2017). Physical performance and positional differences among young female volleyball players. The Journal of sports medicine and physical fitness, 57(10), 1282-1289.

Pereira, A., M Costa, A., Santos, P., Figueiredo, T., \& Vicente João, P. (2015). Training strategy of explosive strength in young female volleyball players. Medicina, 51(2), 126-131.

Reeser, J. C., \& Bahr, R. (2017). Handbook of sports medicine and science, Volleyball: John Wiley \& Sons.

Sahin, H. M. (2014). Relationships between acceleration, agility, and jumping ability in female volleyball players. European Journal of Experimental Biology, 4(1), 303-308. 
Sanders, G. J., Boos, B., Shipley, F., Scheadler, C. M., \& Peacock, C. A. (2018). An Accelerometer-Based Training Load Analysis to Assess Volleyball Performance. Journal of Exercise and Nutrition, 1, 1-4.

Sattler, T., Sekulic, D., Hadzic, V., Uljevic, O., \& Dervisevic, E. (2012). Vertical jumping tests in volleyball: reliability, validity, and playing-position specifics. The Journal of Strength \& Conditioning Research, 26(6), 1532-1538.

Sheppard, J., Hobson, S., Barker, M., Taylor, K., Chapman, D., McGuigan, M., \& Newton, R. (2008). The effect of training with accentuated eccentric load counter-movement jumps on strength and power characteristics of high-performance volleyball players. International Journal of Sports Science \& Coaching, 3(3), 355-363.

Sheppard, J. M., Gabbett, T. J., \& Stanganelli, L.-C. R. (2009). An analysis of playing positions in elite men's volleyball: considerations for competition demands and physiologic characteristics. The Journal of Strength \& Conditioning Research, 23(6), 1858-1866.

Stanganelli, L. C. R., Dourado, A. C., Oncken, P., Mançan, S., \& da Costa, S. C. (2008). Adaptations on jump capacity in Brazilian volleyball players prior to the under-19 World Championship. The Journal of Strength \& Conditioning Research, 22(3), 741-749.

VanHeest, J. L. (2003). Energy demands in the sport of volleyball. Handbook of Sports Medicine and Science: Volleyball, 11-17.

Voelzke, M., Stutzig, N., Thorhauer, H.-A., \& Granacher, U. (2012). Promoting lower extremity strength in elite volleyball players: effects of two combined training methods. Journal of Science and Medicine in Sport, 15(5), 457-462.

Voigt, H.-f., \& Vetter, K. (2003). The value of strength-diagnostic for the structure of jump training in volleyball. European Journal of Sport Science, 3(3), 1-10.

Yingling, V. R., Castro, D. A., Duong, J. T., Malpartida, F. J., Usher, J. R., \& Jenny, O. (2018). The reliability of vertical jump tests between the Vertec and My Jump phone application. PeerJ, 6, e4669. 